\title{
Phonon Dispersion in Amorphous Ni-Alloys
}

\author{
A.M. VORA* \\ Parmeshwari 165, Vijaynagar Area, Hospital Road \\ Bhuj-Kutch, 370 001, Gujarat, India
}

(Received February 6, 2007; revised version April 10, 2007)

\begin{abstract}
The well-known model potential is used to investigate the longitudinal and transverse phonon dispersion curves for six Ni-based binary amorphous alloys, viz. $\mathrm{Ni}_{31} \mathrm{Dy}_{69}, \mathrm{Ni}_{33} \mathrm{Y}_{67}, \mathrm{Ni}_{36} \mathrm{Zr}_{64}, \mathrm{Ni}_{50} \mathrm{Zr}_{50}, \mathrm{Ni}_{60} \mathrm{Nb}_{40}$, and $\mathrm{Ni}_{81} \mathrm{~B}_{19}$. The thermodynamic and elastic properties are also computed from the elastic limits of the phonon dispersion curves. The theoretical approach given by Hubbard-Beeby is used in the present study to compute the phonon dispersion curves. Five local field correction functions proposed by Hartree, Taylor, Ichimaru-Utsumi, Farid et al. and Sarkar et al. are employed to see the effect of exchange and correlation in the aforesaid properties.
\end{abstract}

PACS numbers: 63.50.+x, 65.60.+a

\section{Introduction}

The mankind has been manufacturing glassy materials for several thousands years. Compared to that, the scientific study of amorphous materials has a much shorter history. And only recently there has been an explosion of interest to these studies as more promising materials are produced in the amorphous form. The range of applications of metallic glasses is vast and extends from the common window glass to high capacity storage media for digital devices [1-8].

The $\mathrm{Ni}_{31} \mathrm{Dy}_{69}$ glass is a member of transition metal-lanthanide or actinide (TM-LA) element group, while $\mathrm{Ni}_{81} \mathrm{~B}_{19}$ is a candidate of transition metalmetalloid (TM-M) glass. Another four metallic glasses such as $\mathrm{Ni}_{33} \mathrm{Y}_{67}, \mathrm{Ni}_{36} \mathrm{Zr}_{64}$, $\mathrm{Ni}_{50} \mathrm{Zr}_{50}$, and $\mathrm{Ni}_{60} \mathrm{Nb}_{40}$ are the members of transition metallic (TM-TM) glasses. The phonon dynamics of $\mathrm{Ni}_{33} \mathrm{Y}_{67}$ glass was studied by Arun Pratap et al. [1], by Hubbard-Beeby (HB) [3], and Takeno-Goda (TG) [4] approaches. The vibrational dynamics of $\mathrm{Ni}_{50} \mathrm{Zr}_{50}$ glass has been studied by Gupta et al. [2] using

*e-mail: voraam@yahoo.com 
TG [4] and Bhatia-Singh (BS) [5] approaches. The phonon dynamics of $\mathrm{Ni}_{31} \mathrm{Dy}_{69}$, $\mathrm{Ni}_{36} \mathrm{Zr}_{64}, \mathrm{Ni}_{60} \mathrm{Nb}_{40}$, and $\mathrm{Ni}_{81} \mathrm{~B}_{19}$ metallic glasses is not reported previously using model potential formalism. Recently, we have reported the vibrational properties of $\mathrm{Ca}_{70} \mathrm{Mg}_{30}, \mathrm{Mg}_{70} \mathrm{Zn}_{30}, \mathrm{Pd}_{35} \mathrm{Zr}_{65}$, and $\mathrm{Pd}_{80} \mathrm{Si}_{20}$ metallic glasses [6-8].

Looking to the advantages of metallic glasses, the present paper emphasizes the phonon dynamics of six Ni-based amorphous alloys, viz. $\mathrm{Ni}_{31} \mathrm{Dy}_{69}, \mathrm{Ni}_{33} \mathrm{Y}_{67}$, $\mathrm{Ni}_{36} \mathrm{Zr}_{64}, \mathrm{Ni}_{50} \mathrm{Zr}_{50}, \mathrm{Ni}_{60} \mathrm{Nb}_{40}$, and $\mathrm{Ni}_{81} \mathrm{~B}_{19}$ using the well-known Ashcroft empty core (EMC) model potential [9]. The thermodynamics and elastic properties, such as longitudinal sound velocity $v_{\mathrm{L}}$, transverse sound velocity $v_{\mathrm{T}}$, isothermal bulk modulus $B_{\mathrm{T}}$, modulus of rigidity $G$, Poisson's ratio $\sigma$, Young's modulus $Y$, and Debye temperature $\theta_{\mathrm{D}}$ are computed from the elastic limit of the dispersion relation. Five different types of local field correction functions proposed by Hartree (H) [10], Taylor (T) [11], Ichimaru-Utsumi (IU) [12], Farid et al. (F) [13] and Sarkar et al. (S) [14] are used to study the exchange and correlation effects in the aforesaid studies. The phenomenological theory of HB [3] in the random phase approximation is employed to generate the phonon dispersion curves (PDC). The most important ingredient of the PDC is pair potential computed theoretically in Wills-Harrison (WH) [15] form from EMC potential of Ashcroft [9].

\section{Theoretical methodology}

The fundamental ingredient, which goes into the calculation of the phonon dynamics of metallic glasses, is the pair potential. In the present study, for TM-LA, TM-TM, and TM-M metallic glasses, the pair potential is computed using $[6]$

$V(r)=V_{s}(r)+V_{b}(r)+V_{r}(r)$.

The $s$-electron contribution to the pair potential $V_{s}(r)$ is calculated from

$$
V_{s}(r)=\frac{Z_{s}^{2} e^{2}}{r}+\frac{\Omega_{0}}{\pi^{2}} \int F(q) \frac{\sin (q r)}{q r} q^{2} \mathrm{~d} q .
$$

Here $Z_{s} \approx 1.5$ is found by integrating the partial $s$-density of states resulting from self-consistent band structure calculation for the entire $3 d$ and $4 d$ series [6], while $\Omega_{0}$ is the effective atomic volume of the one component fluid.

The energy wave number characteristics appearing in Eq. (2) is written as [6]

$$
F(q)=-\frac{\Omega_{0} q^{2}}{16 \pi}\left|W_{\mathrm{B}}(q)\right|^{2} \frac{\varepsilon_{\mathrm{H}}(q)-1}{1+\left[\varepsilon_{\mathrm{H}}(q)-1\right][1-f(q)]} .
$$

Here $W_{\mathrm{B}}(q)$ is the effective bare ion potential, $\varepsilon_{\mathrm{H}}(q)$ - the Hartree dielectric response function and $f(q)$ - the local field correction function to introduce the exchange and correlation effects.

The well-known EMC model potential of Ashcroft [9] used in the present computation of phonon dynamics of binary metallic glasses is of the form [9]

$$
W(q)=-\frac{8 \pi Z}{\Omega_{0} q^{2}} \cos \left(q r_{\mathrm{C}}\right),
$$

here $r_{\mathrm{C}}$ is the parameter of the model potential of ternary amorphous alloys. The 
model potential parameter $r_{\mathrm{C}}$ is calculated from the well-known formula [6-8] as follows:

$$
r_{\mathrm{C}}=\frac{0.51 r_{\mathrm{S}}}{Z^{1 / 3}}
$$

Here $r_{\mathrm{S}}$ is the effective Wigner-Seitz radius of the amorphous alloys.

The $d$-electron contributions to the pair potential are expressed in terms of the number of $d$-electron $Z_{d}$, the $d$-state radii $r_{d}$, and the nearest neighbour coordination number $N_{\mathrm{C}}$ as follows:

$$
V_{b}(r)=-Z_{d}\left(1-\frac{Z_{d}}{10}\right)\left(\frac{12}{N_{\mathrm{C}}}\right)^{1 / 2} \frac{28.06}{\pi} \frac{2 r_{d}^{3}}{r^{5}}
$$

and

$$
V_{r}(r)=Z_{d} \frac{450}{\pi^{2}} \frac{r_{d}^{6}}{r^{8}}
$$

The theory for computing the phonon dynamics in amorphous solids, approach proposed by Hubbard-Beeby [3] has been employed in the present computation. The expressions for longitudinal phonon frequency $\omega_{\mathrm{L}}$ and transverse phonon frequency $\omega_{\mathrm{T}}$ per HB approach are $[3,6-8]$ :

$$
\begin{aligned}
& \omega_{\mathrm{L}}^{2}(q)=\omega_{\mathrm{E}}^{2}\left[1-\frac{\sin (q \sigma)}{q \sigma}-\frac{6 \cos (q \sigma)}{(q \sigma)^{2}}+\frac{6 \sin (q \sigma)}{(q \sigma)^{3}}\right], \\
& \omega_{\mathrm{T}}^{2}(q)=\omega_{\mathrm{E}}^{2}\left[1-\frac{3 \cos (q \sigma)}{(q \sigma)^{2}}+\frac{3 \sin (q \sigma)}{(q \sigma)^{3}}\right]
\end{aligned}
$$

with

$$
\omega_{\mathrm{E}}^{2}=\frac{4 \pi \rho}{3 M} \int_{0}^{\infty} g(r) V^{\prime \prime}(r) r^{2} \mathrm{~d} r .
$$

Here $M, \rho$ are the atomic mass and the number density of the glassy alloy, respectively, while $V^{\prime \prime}(r)$ is the second derivative of the pair potential.

In the long wavelength limit of the frequency spectrum, both phonon frequencies viz. the longitudinal $\omega_{\mathrm{L}}$ and transverse $\omega_{\mathrm{T}}$ phonon frequencies are proportional to the wave vectors and obey the relationships [6-8]

$$
\omega_{\mathrm{L}} \propto q \text { and } \omega_{\mathrm{T}} \propto q, \quad \omega_{\mathrm{L}}=v_{\mathrm{L}} q \quad \text { and } \quad \omega_{\mathrm{T}}=v_{\mathrm{T}} q,
$$

where $v_{\mathrm{L}}$ and $v_{\mathrm{T}}$ are the longitudinal and transverse sound velocities of the glassy alloys, respectively.

For HB approach, the formulations for $v_{\mathrm{L}}$ and $v_{\mathrm{T}}$ are given as [3]

and

$$
v_{\mathrm{L}}(\mathrm{HB})=\omega_{\mathrm{E}} \sqrt{\frac{3 \sigma^{2}}{10}}
$$

$$
v_{\mathrm{T}}(\mathrm{HB})=\omega_{\mathrm{E}} \sqrt{\frac{\sigma^{2}}{10}}
$$

The present study also includes isothermal bulk modulus $B_{\mathrm{T}}$, modulus of rigidity $G$, Poisson's ratio $\sigma$, Young's modulus $\mathrm{Y}$, and Debye temperature $\theta_{\mathrm{D}}$ from the elastic limit of the PDC. All the quantities are computed from the longitudinal 
and transverse sound velocities $\left(v_{\mathrm{L}}\right.$ and $\left.v_{\mathrm{T}}\right)$. The bulk modulus $B_{\mathrm{T}}$, modulus of rigidity $G$, Poisson's ratio $\sigma$, Young's modulus $Y$, and Debye temperature $\theta_{\mathrm{D}}$ are obtained using the expressions [6-8],

$$
\begin{aligned}
& B_{\mathrm{T}}=\rho_{\mathrm{M}}\left(v_{\mathrm{L}}^{2}-\frac{4}{3} v_{\mathrm{T}}^{2}\right), \\
& G=\rho_{\mathrm{M}} v_{\mathrm{T}}^{2},
\end{aligned}
$$

where $\rho_{\mathrm{M}}$ is the isotropic number density of the solid,

$$
\begin{aligned}
\sigma & =\frac{1-2 v_{\mathrm{T}}^{2} / v_{\mathrm{L}}^{2}}{2-2 v_{\mathrm{T}}^{2} / v_{\mathrm{L}}^{2}}, \\
Y & =2 G(\sigma+1)
\end{aligned}
$$

and

$$
\theta_{\mathrm{D}}=\frac{\hbar \omega_{\mathrm{D}}}{k_{\mathrm{B}}}=\frac{\hbar}{k_{\mathrm{B}}} 2 \pi\left(\frac{9 \rho}{4 \pi}\right)^{1 / 3}\left(\frac{1}{v_{\mathrm{L}}^{3}}+\frac{2}{v_{\mathrm{T}}^{3}}\right)^{-1 / 3}
$$

Here $\hbar, k_{\mathrm{B}}, \omega_{\mathrm{D}}$ are the Planck constant, Boltzmann constant, and Debye frequency, respectively.

\section{Results and discussion}

The input parameters and other related constants used in the present computations are tabulated in Table I, which are taken from the literature [15].

TABLE I

Input parameters and other constants.

\begin{tabular}{c|c|c|c|c|c|c}
\hline \hline Glasses & $Z$ & $\begin{array}{c}\Omega_{0} \\
\text { [a.u.] }\end{array}$ & $\begin{array}{c}r_{\mathrm{C}} \\
\text { [a.u.] }\end{array}$ & $Z_{d}$ & $N_{\mathrm{C}}$ & $\begin{array}{c}r_{d} \\
\text { [a.u.] }\end{array}$ \\
\hline $\mathrm{Ni}_{31} \mathrm{Dy}_{69}$ & 2.69 & 158.44 & 0.8930 & 3.67 & 12.00 & 2.93 \\
$\mathrm{Ni}_{33} \mathrm{Y}_{67}$ & 2.67 & 160.48 & 0.9028 & 3.81 & 12.00 & 2.44 \\
$\mathrm{Ni}_{36} \mathrm{Zr}_{64}$ & 3.28 & 126.09 & 0.7189 & 4.66 & 12.00 & 2.19 \\
$\mathrm{Ni}_{50} \mathrm{Zr}_{50}$ & 3.00 & 114.47 & 0.7384 & 5.50 & 12.00 & 2.00 \\
$\mathrm{Ni}_{60} \mathrm{Nb}_{40}$ & 3.20 & 90.61 & 0.6632 & 6.50 & 10.40 & 1.77 \\
$\mathrm{Ni}_{81} \mathrm{~B}_{19}$ & 2.19 & 70.77 & 0.7835 & 7.08 & 11.43 & 1.28
\end{tabular}

The presently calculated pair potentials of $\mathrm{Ni}_{31} \mathrm{Dy}_{69}$ glass are shown in Fig. 1a, which elucidates that the inclusion of screening functions hardly changes the nature of the pair potentials, except around the first minimum. The well depth slightly increases due to the influence of various screening functions compared to H-screening. The presently obtained pair potentials of $\mathrm{Ni}_{33} \mathrm{Y}_{67}$ glass are shown in Fig. 1b along with the other such theoretical results $[1,16]$. The first zero position of pair potentials at $r=r_{0}$ due to all screening functions occurs at $r_{0} \approx 3.95$ a.u. Thus the inclusion of exchange and correlation on 

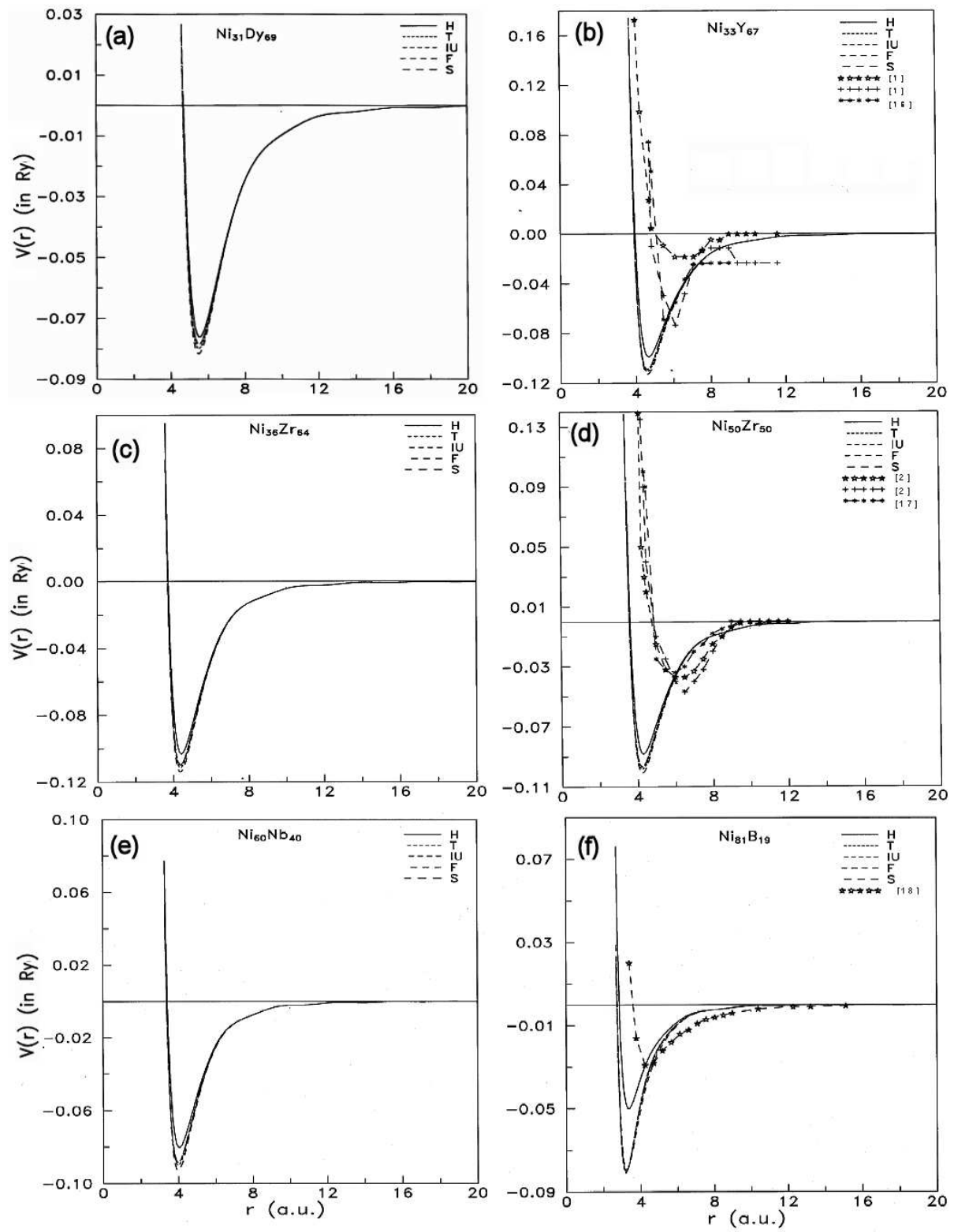

Fig. 1. Pair potentials for (a) $\mathrm{Ni}_{31} \mathrm{Dy}_{69}$, (b) $\mathrm{Ni}_{33} \mathrm{Y}_{67}$, (c) $\mathrm{Ni}_{36} \mathrm{Zr}_{64}$, (d) $\mathrm{Ni}_{50} \mathrm{Zr}_{50}$, (e) $\mathrm{Ni}_{60} \mathrm{Nb}_{40}$, and (f) $\mathrm{Ni}_{81} \mathrm{~B}_{19}$ metallic glasses.

$V\left(r=r_{0}\right)$ is not substantial. But the well width increases compared to H-screening. It is observed that well depth of presently computed pair potentials is shifted towards the left and is also high as compared to the results of Arun Pratap et al. [1] and Hausleitner-Hafner [16]. The presently generated pair po- 
tentials of $\mathrm{Ni}_{36} \mathrm{Zr}_{64}$ glass are displayed in Fig. 1c. The first zero for $V\left(r=r_{0}\right)$ is at $1.0 \AA^{-1}$ point, the influence of $r_{0} \approx 3.74$ a.u. The maximum depth in the pair potentials is obtained for S-function. The pair potentials due to remaining T-, IU-, and F-screening functions are lying between those of $\mathrm{H}$ - and S-screening functions. The presently calculated pair potentials of $\mathrm{Ni}_{50} \mathrm{Zr}_{50}$ glass are observed in Fig. 1d along with the other such available theoretical data $[2,17]$. The position of pair potentials at $r=r_{0}$ due to $\mathrm{H}$-function occurs at $r_{0}=3.62 \mathrm{a} . \mathrm{u}$, while the influence of screenings suppresses this zero slightly and occurs at $r_{0} \leq 3.55$ a.u. The broad well width is seen due to inclusion of local field correction functions. It is seen that well depth of presently computed pair potentials is shifted towards the left and is as high as compared to the outcomes of Gupta et al. [2] and Hausleitner-Hafner [17]. The computed pair potentials of $\mathrm{Ni}_{60} \mathrm{Nb}_{40}$ glass are displayed in Fig. 1e. It is apparent from the behaviour that the inclusion of screening effects increases the well width slightly compared to H-screening. The first zero for $V\left(r=r_{0}\right)$ due to $\mathrm{H}$ occurs at $r_{0}=3.38$ a.u., while inclusion of exchange and correlation suppresses this zero to $r_{0} \leq 3.28$ a.u. The pair potentials of two-component $\mathrm{Ni}_{81} \mathrm{~B}_{19}$ glass are shown in Fig. 1f. It is noticed that the first zero for $V\left(r=r_{0}\right)$ due to all local field correction functions occurs around $r_{0} \approx 3.26$ a.u. The well width also increases with respect to H-screening. The position of $V_{\min }(r)$ is affected by the nature of the screenings. It is observed that well depth of presently computed potentials moves towards the left as compared to that of Hausleitner and Hafner [18]. The present results of pair potentials of all the Ni-based amorphous alloys do not show any oscillatory behaviour and are almost constant in the large $r$-region. The presently computed pair potentials from H-, T-, IU-, F- and S-local field correction functions for most of the amorphous alloys are overlapped with each other.

The pair potentials for the six Ni-based amorphous alloys computed using S-local field correction function is displayed in Fig. 2. In this figure, we have compared our presently computed pair potentials from S-local field correction function with each other for most of the amorphous alloys. This figure indicates the shifting of the pair potentials with respect to atomic volume $\Omega_{0}$ of the amorphous alloys. The repulsive Coulomb interaction and the attractive interactions represented by oscillatory nature are observed. It is also noticed that when volume $\Omega_{0}$ of the glassy alloys increases ( $\Omega_{0}$ is greater for $\mathrm{Ni}_{33} \mathrm{Y}_{67}$ glass), the potential depth deepens. It means that the pair potential for $\mathrm{Ni}_{33} \mathrm{Y}_{67}$ glass shows a higher depth in comparison with other metallic glasses. The potential well width of $\mathrm{Ni}_{81} \mathrm{~B}_{19}$ glass shifts at lower $r$-values, while that of $\mathrm{Ni}_{31} \mathrm{Dy}_{69}$ glass shifts at higher $r$ values. The lanthanide element Dy and metalloid component B plays an important role in the nature of the pair potentials of respective amorphous alloys, i.e. for $\mathrm{Ni}_{31} \mathrm{Dy}_{69}$ glass the repulsive interaction is greater in comparison with $\mathrm{Ni}_{81} \mathrm{~B}_{19}$ metallic glass. All the pair potentials show the combined effect of the $s$ - and $d$-electrons. Bretonnet and Derouiche [19] observed that the repulsive part of $V(r)$ is drawn lower and its attractive part is deeper due to the $d$-electron effect. 




Fig. 2. Pair potentials for $\mathrm{Ni}_{31} \mathrm{Dy}_{69}, \mathrm{Ni}_{33} \mathrm{Y}_{67}, \mathrm{Ni}_{36} \mathrm{Zr}_{64}, \mathrm{Ni}_{50} \mathrm{Zr}_{50}, \mathrm{Ni}_{60} \mathrm{Nb}_{40}$, and $\mathrm{Ni}_{81} \mathrm{~B}_{19}$ metallic glasses using S-local field correction function.

When we go from $\mathrm{Ni}_{31} \mathrm{Dy}_{69} \rightarrow \mathrm{Ni}_{81} \mathrm{~B}_{19}$, the net number of $d$-electron $r_{d}$ decreases, hence the $V(r)$ is shifted towards the lower $r$-values. Therefore, the present results support the $d$-electron effect as noted by Bretonnet and Derouiche [19].

From Figs. 1 and 2, it can be noted that the Coulomb repulsive potential part dominates the oscillations due to ion-electron-ion interactions, which shows the waving shape oscillation of the potential after $r \approx 10$ a.u. Hence, the pair potentials converge towards a finite value instead of zero in repulsive region.

The phonon modes for longitudinal and transverse branches of $\mathrm{Ni}_{31} \mathrm{Dy}_{69}$, $\mathrm{Ni}_{33} \mathrm{Y}_{67}, \mathrm{Ni}_{36} \mathrm{Zr}_{64}, \mathrm{Ni}_{50} \mathrm{Zr}_{50}, \mathrm{Ni}_{60} \mathrm{Nb}_{40}$, and $\mathrm{Ni}_{81} \mathrm{~B}_{19}$ metallic glasses calculated using $\mathrm{HB}$ approach with the five screening functions are shown in Fig. $3 \mathrm{a}-\mathrm{f}$. The phonon eigenfrequencies of $\mathrm{Ni}_{31} \mathrm{Dy}_{69}$ glass calculated using $\mathrm{HB}$ approach to study the screening influence are shown in Fig. 3a. From Fig. 3a, it is seen that the present results of phonon modes due to $\mathrm{H}-$, $\mathrm{T}$-, and $\mathrm{F}$-functions are lying between those due to IU- and S-screenings. The first minimum in the longitudinal branch is found at around $1.72 \AA^{-1}$ for $\mathrm{H}-, 2.36 \AA^{-1}$ for T- and IU-, $2.52 \AA^{-1}$ for F- and S-local field correction functions. The influence of $\mathrm{T}, \mathrm{IU}$, and $\mathrm{F}$ on $\omega_{\mathrm{L}}$ is ranging from $7.9 \%$ to $11 \%$. The S-function enhances the $\omega_{\mathrm{L}}$ of $\mathrm{Ni}_{31} \mathrm{Dy}_{69}$ glass by $\approx 67.8 \%$ in comparison to $\mathrm{H}$-dielectric function. At $q \approx 1.0 \AA^{-1}$ point, the influence of various local field correction functions on $\omega_{\mathrm{T}}$ due to T-, IU-, F-, and S-screening is $19.98 \%, 22.03 \%, 20.27 \%$, and $35.99 \%$, respectively. From Fig. 3b, it is noted that the present results of PDC of $\mathrm{Ni}_{33} \mathrm{Y}_{67}$ glass due to $\mathrm{T}$, IU, and $\mathrm{F}$ are lying between those due to $\mathrm{S}$ - and $\mathrm{H}$-screening. The first depth in the longitudinal branch is observed at around $1.75 \AA^{-1}$ for $\mathrm{H}_{-}, 2.62 \AA^{-1}$ for $\mathrm{T}$ - and IU-, $2.51 \AA^{-1}$ for F-, and $1.74 \AA^{-1}$ for S-function. The screening influence on the first maximum of $\omega_{\mathrm{L}}$ for $\mathrm{T}$ is $47.78 \%$, for IU- is $36.18 \%$, for F- is $46.06 \%$ and for S-screening is $43.14 \%$ with respect to H-screening, which does not include any exchange and correlation effects. 

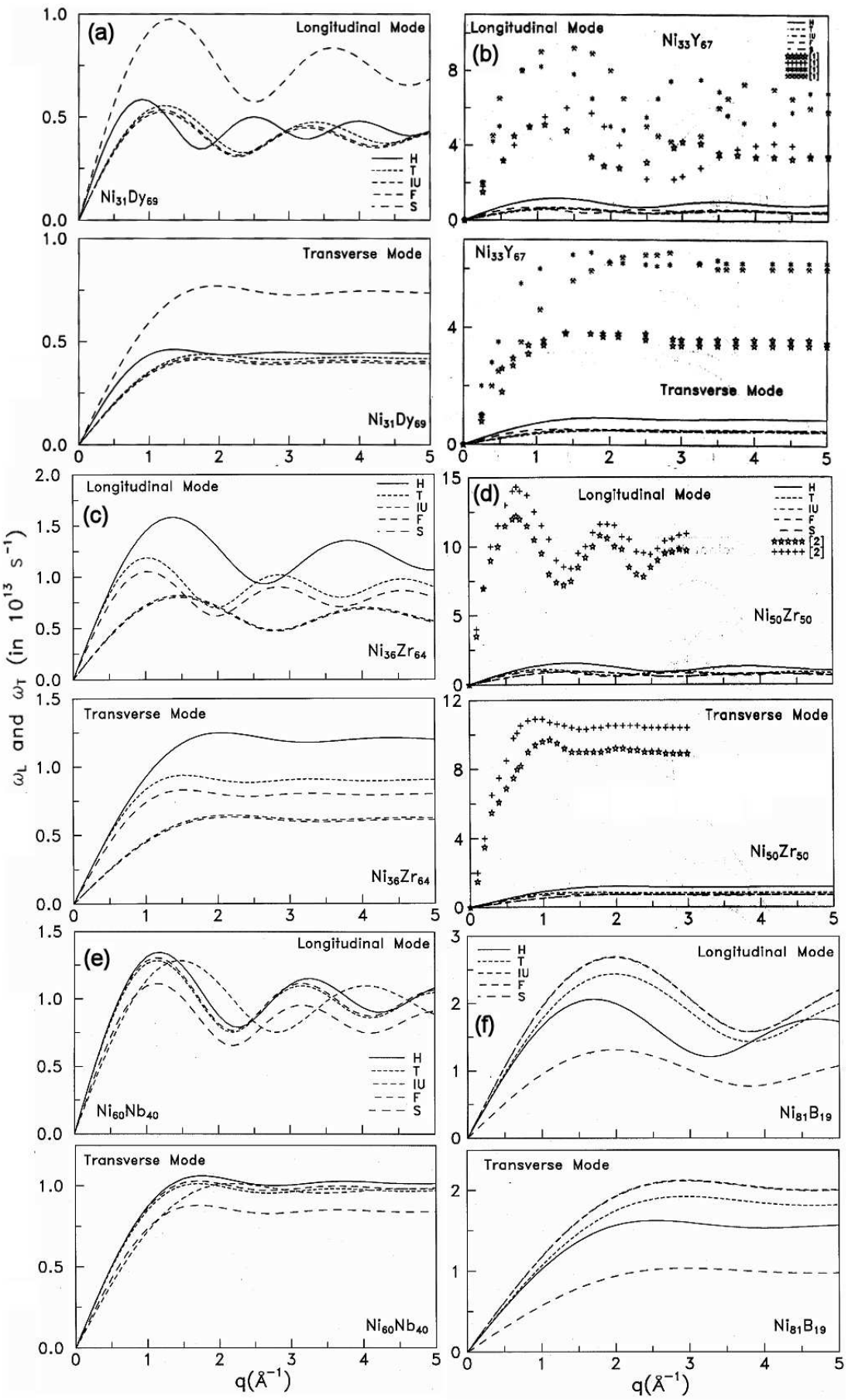

Fig. 3. Phonon dispersion curves for (a) $\mathrm{Ni}_{31} \mathrm{Dy}_{69}$, (b) $\mathrm{Ni}_{33} \mathrm{Y}_{67}$, (c) $\mathrm{Ni}_{36} \mathrm{Zr}_{64}$, (d) $\mathrm{Ni}_{50} \mathrm{Zr}_{50}$, (e) $\mathrm{Ni}_{60} \mathrm{Nb}_{40}$, and (f) $\mathrm{Ni}_{81} \mathrm{~B}_{19}$ metallic glasses. 
At $q \approx 1.0 \AA^{-1}$ point, the influence on $\omega_{\mathrm{T}}$ due to T-, IU-, F-, and S-exchange and correlation functions is $2.75 \%, 17.78 \%, 1.15 \%$, and $15.55 \%$, respectively, in comparison with H-dielectric function. From Fig. 3c, it is evident that the inclusion of exchange and correlation effects suppresses the phonon frequencies of $\mathrm{Ni}_{36} \mathrm{Zr}_{64}$ glass. The first dip in the longitudinal branch is found at around $2.72 \AA^{-1}$ for $\mathrm{H}$-, $2.03 \AA^{-1}$ for $\mathrm{T}$-, $2.85 \AA^{-1}$ for IU- as well as for $\mathrm{F}$-, and $2.02 \AA^{-1}$ for S-screening function. The effect of T-, IU-, F-, and S-screening at first maximum of $\omega_{\mathrm{L}}$ is about $24.91 \%, 67.28 \%, 49.49 \%$, and $33.56 \%$, respectively, in comparison to static H-dielectric function. The screening effect observed at $q \approx 1.0 \AA^{-1}$ position in the transverse branch for T-, IU-, F-, and S-functions is $9.72 \%, 50.47 \%, 51.52 \%$, and $20.18 \%$, respectively. It is noticed from Fig. $3 \mathrm{~d}$ that the inclusion of exchange and correlation effects suppresses the longitudinal as well as transverse phonon branches of $\mathrm{Ni}_{50} \mathrm{Zr}_{50}$ glass. The first dip in the longitudinal branch for $\mathrm{H}-$, T-, IU-, F-, and S-local field correction functions lies at $q \approx 2.72 \AA^{-1}, 2.13 \AA^{-1}$, $2.85 \AA^{-1}, 2.84 \AA^{-1}$, and $2.03 \AA^{-1}$, respectively. The screening influences at first peak of $\omega_{\mathrm{L}}$ with respect to $\mathrm{H}$-screening are $14.73 \%$ for $\mathrm{T}-, 38.46 \%$ for $\mathrm{IU}-, 39.50 \%$ for F-, and $37.08 \%$ for S-screening. Such influence on $\omega_{\mathrm{T}}$ at $q \approx 1.0 \AA^{-1}$ for $\mathrm{T}$ - is $14.17 \%$, for IU- is $41.10 \%$, for $\mathrm{F}$ - is $42.11 \%$, and for S-screening is $24.21 \%$. It is evident from Fig. 3e that the height of the first peak and the position of the first peak in the longitudinal and transverse modes of $\mathrm{Ni}_{60} \mathrm{Nb}_{40}$ glass are appreciably influenced by different screenings. The first minimum in the longitudinal branch for H-, T-, IU-, F-, and S-local field correction functions occurs at $q \approx 2.32 \AA^{-1}$, $2.22 \AA^{-1}, 2.21 \AA^{-1}, 2.36 \AA^{-1}$, and $2.05 \AA^{-1}$, respectively. The effect of the T-screening at the first peak of $\omega_{\mathrm{L}}$ is $3.65 \%$ with respect to $\mathrm{H}$-function. Such effect of IU-, F-, and S-function is $3.65 \%, 2.10 \%$, and $16.44 \%$, respectively. The screening influence on $\omega_{\mathrm{T}}$ at $q \approx 1.0 \AA^{-1}$ point due to T-dielectric function is $2.71 \%$, due to IU- is $17.75 \%$, due to F-function is $1.10 \%$ and due to S- is $15.58 \%$ with respect to $\mathrm{H}$-dielectric function. It is seen from Fig. 3f that the inclusion of exchange and correlation effects enhances the phonon frequencies of $\mathrm{Ni}_{81} \mathrm{~B}_{19}$ glass in longitudinal as well as transverse branches except for $f(q)$ of S-function. The first minimum in the longitudinal branch for H-, T-, IU-, F-, and S-local field correction functions is at around $q \approx 3.35 \AA^{-1}, 3.32 \AA^{-1}, 3.31 \AA^{-1}, 3.34 \AA^{-1}$, and $3.45 \AA^{-1}$, respectively. At first maximum, the screening influence on $\omega_{\mathrm{L}}$ is $0.48 \%$ to $11.25 \%$ with respect to static H-screening while such influence on $\omega_{\mathrm{T}}$ at $q \approx 1.0 \AA^{-1}$ point is about $14.78 \%$ to $63.10 \%$.

The results for the PDC obtained using the S-local field correction function are shown in Fig. 4. It is observed from Fig. 4 that the first peak position of longitudinal branch of $\mathrm{Ni}_{31} \mathrm{Dy}_{69}$ glass is higher while that of $\mathrm{Ni}_{33} \mathrm{Y}_{67}$ glass is lower in comparison with other metallic glasses. Also the first minima of longitudinal branch of $\mathrm{Ni}_{81} \mathrm{~B}_{19}$ glass are seen at higher $q$-values, while for $\mathrm{Ni}_{33} \mathrm{Y}_{67}$ glass at lower $q$-values. The lower dip in the longitudinal branch justifies the correctness and stability of the pair potential. The same results are observed in the transverse 


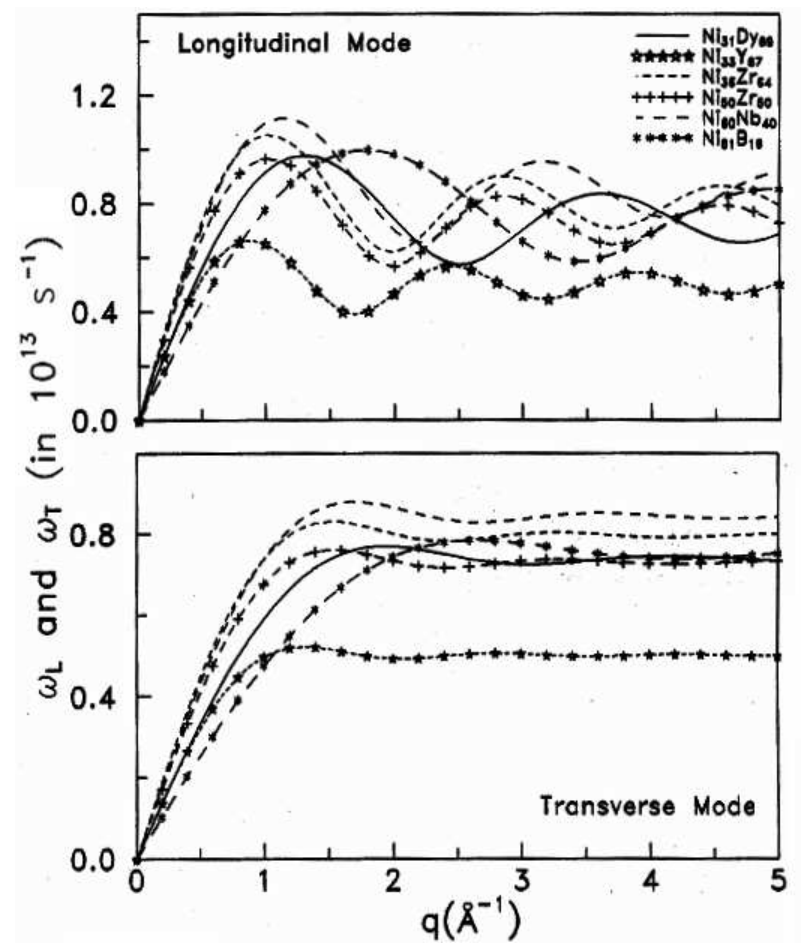

Fig. 4. Phonon dispersion curves for $\mathrm{Ni}_{31} \mathrm{Dy}_{69}, \mathrm{Ni}_{33} \mathrm{Y}_{67}, \mathrm{Ni}_{36} \mathrm{Zr}_{64}, \mathrm{Ni}_{50} \mathrm{Zr}_{50}, \mathrm{Ni}_{60} \mathrm{Nb}_{40}$, and $\mathrm{Ni}_{81} \mathrm{~B}_{19}$ metallic glasses using S-local field correction function.

branch, too. Moreover, it is observed from Fig. 4 that the oscillations are more prominent in the longitudinal phonon modes as compared to the transverse modes. This shows the existence of collective excitations at larger momentum transfer due to longitudinal phonons only and the instability of the transverse phonons due to the anharmonicity of the atomic vibrations in the metallic systems. Also in the high wave vector region, damping of phonons dominates the transverse mode which is indicating the fluid characteristics of the glass, i.e. transverse phonon behaviour is monotonic. Here in the transverse branch, the frequencies increase with wave vector $q$ and then saturate at $q \approx 2.0 \AA^{-1}$, which supports the well-known Thorpe model [20] in which a glass like a solid containing finite liquid cluster is described. The transverse phonons are absorbed for frequencies larger than the smallest eigenfrequencies of the largest cluster.

Furthermore, the thermodynamic and elastic properties of Ni-based amorphous alloys estimated from the elastic limit of the PDC are tabulated in Table II. The present results of $\mathrm{Ni}_{33} \mathrm{Y}_{67}$ glass are found lower in line with other theoretical data [1]. Also the present outcome of $\mathrm{Ni}_{50} \mathrm{Zr}_{50}$ glass shows lower results in comparison with the theoretical reported data [2]. The inclusion of various local field corrections with static H-local field correction function is found 
TABLE II

Thermodynamic and elastic properties of amorphous Ni-based alloys. MG means metallic glass, Scr means screening function.

\begin{tabular}{|c|c|c|c|c|c|c|c|c|}
\hline$\overline{\mathrm{MG}}$ & 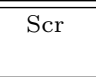 & $\begin{array}{c}v_{\mathrm{L}} \times 10^{5} \\
{[\mathrm{~cm} / \mathrm{s}]}\end{array}$ & $\begin{array}{c}v_{\mathrm{T}} \times 10^{5} \\
{[\mathrm{~cm} / \mathrm{s}]}\end{array}$ & $\begin{array}{c}B_{\mathrm{T}} \times 10^{11} \\
{\left[\text { dyne } / \mathrm{cm}^{2}\right]}\end{array}$ & $\begin{array}{c}G \times 10^{11} \\
{\left[\text { dyne } / \mathrm{cm}^{2}\right]}\end{array}$ & $\overline{\sigma \sigma}$ & $\begin{array}{c}Y \times 10^{11} \\
{\left[\text { dyne } / \mathrm{cm}^{2}\right]}\end{array}$ & $\begin{array}{l}\theta_{\mathrm{D}} \\
{[\mathrm{K}]}\end{array}$ \\
\hline \multirow{5}{*}{$\mathrm{Ni}_{31} \mathrm{Dy}_{69}$} & $\mathrm{H}$ & 1.04 & 0.60 & 0.55 & 0.33 & 0.25 & 0.83 & 69.33 \\
\hline & $\mathrm{T}$ & 0.73 & 0.42 & 0.27 & 0.16 & 0.25 & 0.41 & 48.72 \\
\hline & IU & 0.71 & 0.41 & 0.26 & 0.16 & 0.25 & 0.39 & 47.43 \\
\hline & $\mathrm{F}$ & 0.73 & 0.42 & 0.27 & 0.16 & 0.25 & 0.41 & 48.50 \\
\hline & $\mathrm{S}$ & 1.20 & 0.69 & 0.74 & 0.44 & 0.25 & 1.10 & 79.92 \\
\hline \multirow{9}{*}{$\mathrm{Ni}_{33} \mathrm{Y}_{67}$} & $\mathrm{H}$ & 1.49 & 0.86 & 0.68 & 0.41 & 0.25 & 1.01 & 98.60 \\
\hline & $\mathrm{T}$ & 0.73 & 0.42 & 0.17 & 0.10 & 0.25 & 0.25 & 48.79 \\
\hline & IU & 0.83 & 0.48 & 0.21 & 0.13 & 0.25 & 0.32 & 55.08 \\
\hline & $\mathrm{F}$ & 0.77 & 0.45 & 0.18 & 0.11 & 0.25 & 0.27 & 51.29 \\
\hline & S & 1.21 & 0.70 & 0.45 & 0.27 & 0.25 & 0.67 & 80.16 \\
\hline & & 5.00 & 3.61 & 4.18 & \multirow{4}{*}{-} & \multirow{4}{*}{-} & \multirow{4}{*}{-} & \multirow{4}{*}{-} \\
\hline & Others & 4.95 & 3.20 & 6.44 & & & & \\
\hline & {$[1]$} & 5.11 & 3.14 & 7.12 & & & & \\
\hline & & 4.60 & 2.70 & 6.79 & & & & \\
\hline \multirow{5}{*}{$\mathrm{Ni}_{36} \mathrm{Zr}_{64}$} & $\mathrm{H}$ & 1.84 & 1.06 & 1.25 & 0.75 & 0.25 & 1.87 & 132.51 \\
\hline & $\mathrm{T}$ & 1.85 & 1.07 & 1.26 & 0.76 & 0.25 & 1.89 & 133.34 \\
\hline & IU & 0.90 & 0.52 & 0.30 & 0.18 & 0.25 & 0.45 & 64.63 \\
\hline & $\mathrm{F}$ & 0.88 & 0.51 & 0.28 & 0.17 & 0.25 & 0.43 & 63.30 \\
\hline & S & 1.64 & 0.95 & 0.99 & 0.59 & 0.25 & 1.48 & 117.98 \\
\hline \multirow{8}{*}{$\mathrm{Ni}_{50} \mathrm{Zr}_{50}$} & $\mathrm{H}$ & 1.78 & 1.03 & 1.29 & 0.78 & 0.25 & 1.94 & 132.33 \\
\hline & $\mathrm{T}$ & 1.70 & 0.98 & 1.18 & 0.71 & 0.25 & 1.78 & 126.62 \\
\hline & IU & 1.03 & 0.60 & 0.44 & 0.26 & 0.25 & 0.65 & 76.89 \\
\hline & $\mathrm{F}$ & 1.02 & 0.59 & 0.42 & 0.25 & 0.25 & 0.63 & 75.60 \\
\hline & S & 1.50 & 0.87 & 0.92 & 0.55 & 0.25 & 1.38 & 111.69 \\
\hline & & & & & & & & 370.50 \\
\hline & Others & 9.11 & 6.58 & 1.74 & - & - & - & 419.90 \\
\hline & {$[2]$} & 9.53 & 6.96 & 1.80 & & & & 443.77 \\
\hline \multirow{5}{*}{$\mathrm{Ni}_{60} \mathrm{Nb}_{40}$} & $\mathrm{H}$ & 1.83 & 1.06 & 1.67 & 1.00 & 0.25 & 2.50 & 147.03 \\
\hline & $\mathrm{T}$ & 1.80 & 1.04 & 1.62 & 0.97 & 0.25 & 2.42 & 144.81 \\
\hline & IU & 1.41 & 0.81 & 0.99 & 0.59 & 0.25 & 1.48 & 113.21 \\
\hline & $\mathrm{F}$ & 1.83 & 1.06 & 1.67 & 1.00 & 0.25 & 2.50 & 147.21 \\
\hline & S & 1.57 & 0.90 & 1.22 & 0.73 & 0.25 & 1.83 & 125.75 \\
\hline \multirow{5}{*}{$\mathrm{Ni}_{81} \mathrm{~B}_{19}$} & $\mathrm{H}$ & 1.95 & 1.12 & 1.65 & 0.99 & 0.25 & 2.48 & 169.68 \\
\hline & $\mathrm{T}$ & 1.98 & 1.14 & 1.71 & 1.03 & 0.25 & 2.56 & 172.69 \\
\hline & IU & 2.19 & 1.26 & 2.09 & 1.25 & 0.25 & 3.13 & 190.72 \\
\hline & $\mathrm{F}$ & 2.18 & 1.26 & 2.07 & 1.24 & 0.25 & 3.11 & 190.05 \\
\hline & S & 1.07 & 0.62 & 0.50 & 0.30 & 0.25 & 0.74 & 92.95 \\
\hline
\end{tabular}

between $15.28 \%-53.26 \%, 18.85 \%-72.88 \%, 0.68 \%-77.18 \%, 4.38 \%-67.45 \%, 1.68 \%-$ $40.87 \%$, and $1.88 \%-70.10 \%$ for $\mathrm{Ni}_{31} \mathrm{Dy}_{69}, \mathrm{Ni}_{33} \mathrm{Y}_{67}, \mathrm{Ni}_{36} \mathrm{Zr}_{64}, \mathrm{Ni}_{50} \mathrm{Zr}_{50}, \mathrm{Ni}_{60} \mathrm{Nb}_{40}$, and $\mathrm{Ni}_{81} \mathrm{~B}_{19}$ metallic glasses, respectively. It is seen that the screening theory plays an important role in the prediction of the thermodynamic and elastic properties of metallic glasses. The present values of the isothermal bulk modulus $B_{\mathrm{T}}$ change with the atomic volume of binary metallic glass which shows a close similarity in the response of crystalline and disordered structures to compressive stains. The present results of sound velocities of $\mathrm{Ni}_{33} \mathrm{Y}_{67}$ and $\mathrm{Ni}_{50} \mathrm{Zr}_{50}$ binary metal- 
lic glasses are more than five and nine times lower than the highest theoretical data $[1,2]$. The percentile influences of longitudinal sound velocity $v_{\mathrm{L}}$ of $\mathrm{Ni}_{33} \mathrm{Y}_{67}$ and $\mathrm{Ni}_{50} \mathrm{Zr}_{50}$ binary metallic glasses with respect to the available highest theoretical data $[1,2]$ are found around $70.84 \%-85.71 \%$ and $80.46 \%-88.80 \%$, respectively, while those influences of transverse sound velocity $v_{\mathrm{T}}$ are found around $76.18 \%-$ $86.70 \%$ and $89.37 \%-93.91 \%$. The percentile influences of isothermal bulk modulus $B_{\mathrm{T}}$ of $\mathrm{Ni}_{33} \mathrm{Y}_{67}$ and $\mathrm{Ni}_{50} \mathrm{Zr}_{50}$ binary metallic glasses with respect to the available highest theoretical data $[1,2]$ are found around $90.45 \%-91.61 \%$ and $28.33 \%-$ $76.63 \%$, respectively.

The dielectric function plays an important role in the evaluation of potential due to the screening of the electron gas. For this purpose in the present investigation, the local field correction functions due to $\mathrm{H}, \mathrm{T}, \mathrm{IU}, \mathrm{F}$, and $\mathrm{S}$ are used. The reason for selecting these functions is that $\mathrm{H}$-function does not include exchange and correlation effect and represents only static dielectric function, while T-function covers the overall features of the various local field correction functions proposed before 1972. IU-, F-, and S-functions are the most recent ones among the existing functions and not exploited rigorously in such study. This helps us to study the relative effects of exchange and correlation in the aforesaid properties. Hence, the five different local field correction functions show variations up to an order of magnitude in all the properties.

\section{Conclusions}

Lastly it is concluded that in the study of phonon dynamics of metallic glasses the pair potentials and its derivatives as well as pair correlation function play an important role. In the present computation, the $\mathrm{WH}$ form is adopted to generate the pair potentials, which ignores the angular interaction due to partially filled $d$-bands in transition metals. The well-known model potential with $\mathrm{WH}$ model and HB approach produces consistent results of phonon dynamics for all the metallic glasses. Hence the present EMC model potential is suitable for studying the phonon dynamics of six Ni-based metallic glasses, which confirms applicability of the model potential in the aforementioned study. Such study on phonon dynamics of other binary as well as ternary liquid alloys and metallic glasses is in progress, which will be communicated in the near future.

\section{References}

[1] Arun Pratap, Deepika Bhandari, N.S. Saxena, M.P. Saksena, Ind. J. Pure Appl. Phys. 32, 128 (1994).

[2] N. Gupta, K.C. Jain, N.S. Saxena, Phys. Status Solidi B 176, 81 (1993).

[3] J. Hubbard, J.L. Beeby, J. Phys. C, Solid State Phys. 2, 556 (1969).

[4] S. Takeno, M. Goda, Prog. Theor. Phys. 45, 331 (1971); Prog. Theor. Phys. 47, 790 (1972).

[5] A.B. Bhatia, R.N. Singh, Phys. Rev. B 31, 4751 (1985). 
[6] A.M. Vora, J. Non-Cryst. Solids 352, 3217 (2006).

[7] A.M. Vora, Chinese Phys. Lett. 23, 1872 (2006).

[8] A.M. Vora, J. Mater. Sci. 42, 935 (2007).

[9] N.W. Ashcroft, Phys. Lett. 23, 48 (1966).

[10] W.A. Harrison, Elementary Electronic Structure, World Scientific, Singapore 1999.

[11] R. Taylor, J. Phys. F, Met. Phys. 8, 1699 (1978).

[12] S. Ichimaru, K. Utsumi, Phys. Rev. B 24, 7385 (1981).

[13] B. Farid, V. Heine, G. Engel, I.J. Robertson, Phys. Rev. B 48, 11602 (1993).

[14] A. Sarkar, D.S. Sen, S. Haldar, D. Roy, Mod. Phys. Lett. B 12, 639 (1998).

[15] J.M. Wills, W.A. Harrison, Phys. Rev. B 28, 4363 (1983).

[16] Ch. Hausleitner, J. Hafner, J. Phys., Condens. Matter 2, 6651 (1990).

[17] Ch. Hausleitner, J. Hafner, Phys. Rev. B 42, 5683 (1990).

[18] Ch. Hausleitner, J. Hafner, Phys. Rev. B 47, 5689 (1993).

[19] J.L. Bretonnet, A. Derouiche, Phys. Rev. B 43, 8924 (1990).

[20] M.F. Thorpe, J. Non-Cryst. Solids 57, 355 (1983). 